\title{
Clinical features of spinal schwannomas in 65 patients with schwannomatosis compared with 831 with solitary schwannomas and 102 with neurofibromatosis Type 2: a retrospective study at a single institution
}

\author{
Peng Li, MD, ${ }^{1}$ Fu Zhao, MD, ${ }^{2}$ Jing Zhang, BE, ${ }^{2}$ Zhenmin Wang, MD, ${ }^{1}$ Xingchao Wang, MD, ${ }^{1}$ \\ Bo Wang, MD, ${ }^{1}$ Zhijun Yang, MD, ${ }^{1}$ Jun Yang, MD, ${ }^{1}$ Zhixian Gao, MD, ${ }^{1}$ and Pinan Liu, MD1
}

'Department of Neurosurgery, Beijing Tian Tan Hospital, Capital Medical University, Beijing, China; and 2Department of Neural Reconstruction, Beijing Neurosurgery Institute, Capital Medical University, Beijing, China

\begin{abstract}
OBJECTIVE The aim of this study was to evaluate the clinical features of spinal schwannomas in patients with schwannomatosis and compare them with a large cohort of patients with solitary schwannomas and neurofibromatosis Type 2 (NF2).

METHODS The study was a retrospective review of 831 patients with solitary schwannomas, 65 with schwannomatosis, and 102 with NF2. The clinical, radiographic, and pathological data were extracted with specific attention to the age at onset, location of tumors, initial symptoms, family history, and treatment outcome.
\end{abstract}

RESULTS The male-to-female ratio of patients with schwannomatosis ( $72.3 \%$ vs $27.7 \%$ ) was significantly higher than that of patients with solitary schwannomas ( $53.3 \%$ vs $46.7 \%$ ) and NF2 (54.0\% vs $46.0 \%)$, respectively (chi-square test, $p=0.012)$. The mean age at the first spinal schwannoma operation of patients with NF2 (24.7 \pm 10.2 years) was significantly younger than that of patients with solitary schwannomas (44.8 \pm 13.2 years) and schwannomatosis (44.4 \pm 14.1 years; 1 -way ANOVA, $p<0.001$ ). The initial symptoms were similar among the 3 groups, with pain being the most common. The distribution of spinal tumors among the 3 groups was significantly different. The peak locations of spinal schwannomas in patients with solitary schwannomas were at C1-3 and T12-L3; in schwannomatosis, the peak location was at T12-L5. A preferred spinal location was not evident for intradural-extramedullary tumors in NF2. Only a slight prominence in the lumbar area could be observed. The patients in the 3 groups obtained similar benefits from the operation; the recovery rates in the patients with solitary schwannomas, NF2, and schwannomatosis were $50.1 \%, 38.0 \%$, and $53.9 \%$, respectively. The prognosis varied among spinal schwannomas in the patients with schwannomatosis. Up until the last date of follow-up, most patients with schwannomatosis (81.5\%) had undergone a single spinal operation, but 12 patients $(18.5 \%)$ had undergone multiple spinal operations. Patients with nonsegmental schwannomatosis or those with early onset disease seemed to have a poor prognosis; they were more likely to undergo multiple spinal operations. Small cauda equina nodules were common in patients with schwannomatosis (46.7\%) and NF2 (86.9\%); these small schwannomas appeared to have relatively static behavior. Two patients suspicious for schwannomatosis were diagnosed with NF2 with the detection of constitutional NF2 mutations; 1 had unilateral vestibular schwannoma, and the other had suspicious bilateral trigeminal schwannomas.

CONCLUSIONS The clinical features of spinal schwannomas vary among patients with solitary schwannomas, NF2, and schwannomatosis. Spinal schwannomas of patients with NF2 appear to be more aggressive than those in patients with solitary schwannomas and schwannomatosis. Spinal schwannomas of schwannomatosis predominate in the lumbar area, and most of them can be treated successfully with surgery. The prognosis varies among spinal schwannomas of schwannomatosis; some patients may need multiple operations due to newly developed schwannomas. Sometimes, it is difficult to differentiate schwannomatosis from NF2 based on clinical manifestations. It is prudent to perform close followup examinations in patients with undetermined schwannomatosis and their offspring.

http://thejns.org/doi/abs/10.3171/2015.3.SPINE141145

KEY WORDS clinical features; neurofibromatosis Type 2; oncology; spinal schwannoma; schwannomatosis

ABBREVIATIONS NF = neurofibromatosis; NF1 = NF Type 1; NF2 = NF Type 2; TS = trigeminal schwannoma.

SUBMITTED November 8, 2014. ACCEPTED March 26, 2015.

INCLUDE WHEN CITING Published online September 25, 2015; DOI: 10.3171/2015.3.SPINE141145. 
$\mathrm{S}$ CHWANNOMATOSIS, characterized by a predisposition to develop multiple schwannomas, is a newly recognized third form of neurofibromatosis. Unlike neurofibromatosis Type 2 (NF2), vestibular schwannomas and other types of tumors are rarely observed in schwannomatosis. ${ }^{35}$ Schwannomatosis has only been recognized for the past 2 decades. Its annual incidence is reported to be 0.58 cases per $1,000,000$ persons. $^{2}$

Because the schwannomatosis phenotype overlaps with NF2, schwannomatosis was initially thought to be a mild form of NF2. In 1997, the first research criteria for schwannomatosis were proposed. ${ }^{17}$ Patients with 2 or more pathologically confirmed schwannomas and who lacked radiographic evidence of vestibular schwannoma on an imaging study performed after the age of 18 years were now diagnosed with schwannomatosis. It was not until 2003 that the NF2 locus was excluded as the cause of familial schwannomatosis. Additionally, it was clear that schwannomatosis was clinically and genetically distinct from NF2. ${ }^{24}$ In 2005 and 2006, consensus criteria and revised clinical criteria for schwannomatosis were proposed. ${ }^{3,23}$ These 2 criteria were much more critical than the previous research criteria. The revised clinical criteria excluded patients who fulfilled any existing NF2 diagnostic criteria or had known constitutional NF2 mutations. According to the consensus criteria and revised clinical criteria, patients with 2 or more nonintradermal schwannomas (at least 1 with pathological confirmation) and who lacked a vestibular schwannoma on high-quality MRI performed after the age of 30 years could be diagnosed with schwannomatosis. For many individuals presenting with schwannomatosis without eighth cranial nerve symptoms in the 4th or 5th decade (well past the average age of onset of hearing loss due to NF2 in the 2nd decade), it may be both impractical and clinically irrelevant to obtain a high-resolution head MR image. ${ }^{23}$ Thus, according to the consensus criteria, patients with 2 or more nonintradermal schwannomas and who lacked the symptoms of eighth cranial nerve dysfunction after the age of 45 years could be diagnosed as having presumptive schwannomatosis. Because no NIH guidelines for the diagnosis of schwannomatosis have been created, these 3 criteria have now been used most often.

As schwannomatosis has only been recognized recently, and its incidence is quite low, its clinical features are not well known. Merker et al. ${ }^{26}$ recently reported the largest cohort of 87 patients with schwannomatosis. Although spinal schwannomas are common in schwannomatosis (74\% as reported ${ }^{26}$ ), and they are more likely to cause functional disabilities than peripheral schwannomas, their clinical features have not been fully studied except for several cases or small series reports..$^{8,13,15,22,33}$ In this report, we first describe the detailed clinical features of spinal schwannomas in schwannomatosis and compare them with solitary schwannomas and NF2 in a large cohort of patients.

\section{Methods}

The clinical records of patients with pathologically confirmed spinal schwannomas examined at Beijing Tian
Tan Hospital between January 2008 and August 2014 were retrospectively reviewed. The clinical, radiographic, and pathological data were extracted with specific attention to the age at onset, location of tumors, initial symptoms, family history, other stigmata of neurofibromatosis Type 1 (NF1) or NF2, and treatment outcome. If possible, blood samples of patients with schwannomatosis were analyzed to exclude constitutional NF2 mutations. These patients were followed up through telephone interviews or an outpatient department visit to obtain more detailed medical information. This retrospective study was approved by the Beijing Tian Tan Hospital institutional review board. Informed consent was obtained from all individuals donating tissue for this work.

Patients with a single spinal schwannoma and without other stigmata of NF1 or NF2 were classified as patients with solitary schwannomas. Patients who fulfilled the Manchester criteria for diagnosis of NF2 or had constitutional NF2 mutations were diagnosed with NF2. ${ }^{11}$ In our study, patients who fulfilled the following criteria were classified as having schwannomatosis: 1) 2 or more pathologically confirmed nonintradermal schwannomas; 2) no fulfillment of any of the existing diagnostic criteria for NF2; 3) no first-degree relative with NF2; 4) patients older than 18 years of age; 5) patients younger than 30 years of age with blood samples analyzed to exclude constitutional NF2 mutations; 6) patients under 45 years of age with high-quality enhanced cranial MR images to exclude vestibular schwannomas; and 7) patients older than 45 years of age without symptoms of eighth nerve or other cranial nerve dysfunction.

Mutation analysis was performed on genomic DNA extracted from blood lymphocytes. Polymerase chain reaction (PCR) amplification of NF2 exons 1-17 was performed using oligonucleotide primers designed from the gene sequence to include the whole exon and flanking intronic sequence. PCR products were analyzed by direct bidirectional sequencing using the ABI Prism 3100 sequence analyzer. Multiplex ligation-dependent probe amplification analysis (MRC Holland) was used to detect genomic rearrangements in the coding as well as in the promoter region of NF2 on DNA samples.

For statistical data analysis, the Student independent ttest was used for normally distributed data. We conducted a Kolmogorov-Smirnov test to verify data distribution. One-way ANOVA and post hoc Scheffe tests were used for data with more than 2 groups. For nonnumerical data, the chi-square test was used. A p value $<0.05$ was considered statistically significant. Sometimes the authors performed the chi-square test 4 times in 1 analysis; a $p$ value $<0.013$ was considered statistically significant. The analyses were performed using SPSS statistical software (version 19, IBM).

\section{Results}

\section{Patient Groups}

Between January 2008 and August 2014, 951 patients underwent surgery for spinal schwannomas at our institution; 831 were patients with solitary schwannomas, 50 were patients with NF2, and 70 were patients with pre- 
sumptive schwannomatosis. Additionally, 38 patients with NF2 who underwent whole-spine MRI and 14 who underwent lumbar spine MRI were also included in the cohort to analyze the distribution of spinal tumors, although they did not undergo surgery for spinal schwannomas. As a result, a total of 102 patients with NF2 were included in our cohort.

Of the total 70 patients with presumptive schwannomatosis, 65 met the inclusion criteria of schwannomatosis for our study. The other 5 patients were excluded although they fulfilled the research criteria ${ }^{17}$ for schwannomatosis for the following reasons: blood samples of 2 patients under 30 years of age were not available, and 3 patients under 45 years of age had not undergone cranial MR imaging. Of the 65 patients with schwannomatosis, 51 had highquality cranial MR images. The remaining 14 patients without a cranial MR image were all older than 45 years of age, and they did not have symptoms of eighth nerve or other cranial nerve dysfunction. The blood samples of 13 patients were available to exclude constitutional NF2 mutations. All 5 patients under 30 years of age did not have constitutional NF2 mutations.

\section{Patient Characteristics}

The male-to-female ratio of patients with schwannomatosis $(72.3 \%$ vs $27.7 \%$ ) was significantly higher than that of patients with solitary schwannomas (53.3\% vs $46.7 \%)$ and NF2 (54.0\% vs 46.0\%), respectively (chi-square test, p $=0.012$; Fig. 1B). The mean age at the first spinal schwannoma operation in patients with NF2 $(24.7 \pm 10.2$ years) was significantly younger than that of patients with solitary schwannomas $(44.8 \pm 13.2$ years $)$ and schwannoma- tosis (44.4 \pm 14.1 years; 1 -way ANOVA, $\mathrm{p}<0.001$; Fig. $1 \mathrm{~A}$ and $\mathrm{C}$ ). The median duration of preoperative symptoms in NF2 patients (6 months; range 1 month-5 years) was shorter than that in patients with solitary schwannomas (12 months; range 0.5 months-22 years) and schwannomatosis (18 months; range 1 month-10 years).

\section{Initial Symptoms and Spinal Distribution}

The initial symptoms among the 3 groups were different, but not statistically significantly different (chi-square test, $\mathrm{p}=0.209$; Fig. 2 upper). In 831 patients with solitary schwannomas, the most common initial symptom was pain $(69.5 \%)$ followed by paresthesia/numbness $(35.5 \%)$, motor weakness $(21.7 \%)$, and others (4.8\%). The "others" category included voiding difficulty, gait abnormalities, dizziness, fasciculations, and dysphagia. Because 1 patient could have multiple initial symptoms, the sum of the proportion of different initial symptoms was greater than $100 \%$. In 50 patients with NF2 who underwent surgery, the most common initial symptom was pain $(52.0 \%)$ followed by paresthesia/numbness $(40.0 \%)$, motor weakness $(22.0 \%)$, and others (12.0\%). In 65 patients with schwannomatosis, the most common initial symptom was pain $(64.6 \%)$ followed by paresthesia/numbness $(27.7 \%)$, motor weakness $(24.6 \%)$, and others $(4.5 \%)$. The initial symptoms could vary depending on the spinal location of the schwannomas; the most common initial symptom in the cervical area was paresthesia/numbness instead of pain (Fig. 2 lower).

The distribution of spinal tumors among the 3 groups varied significantly (chi-square test, $\mathrm{p}<0.001$; solitary vs schwannomatosis, $\mathrm{p}<0.001$; schwannomatosis vs NF2,
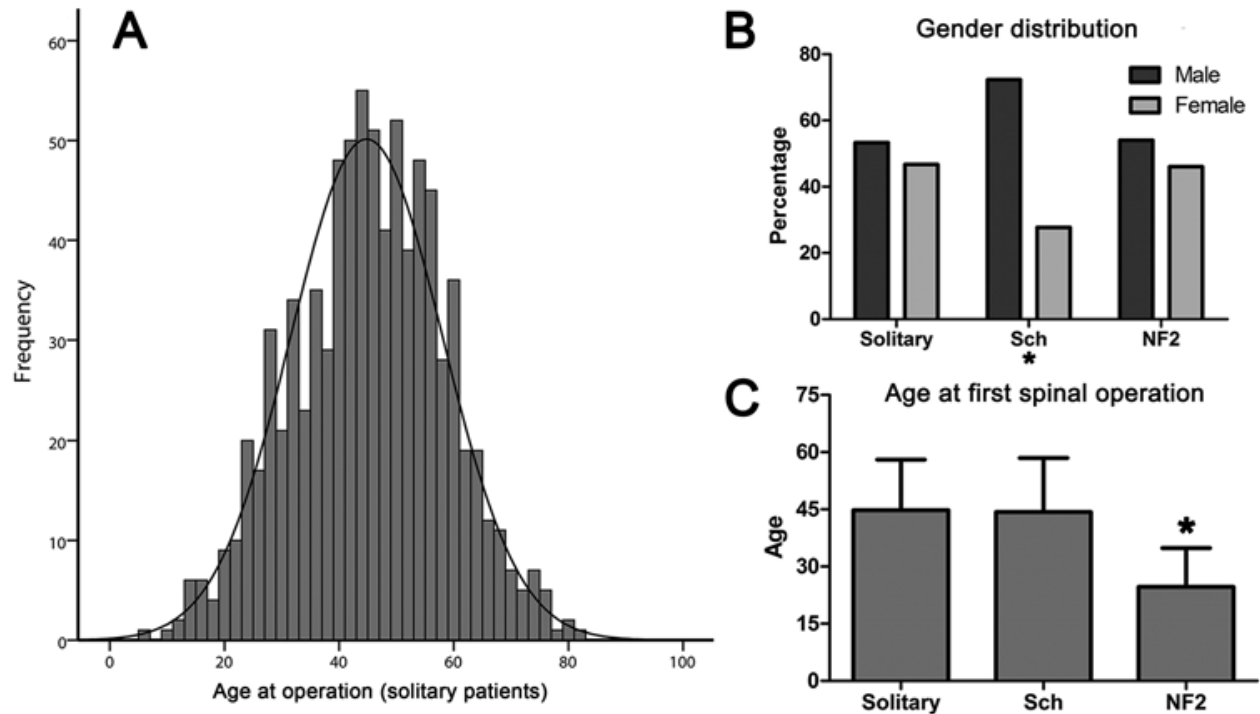

FIG. 1. Bar graphs depicting sex and age distribution in the 3 groups. A: Distribution of age at operation in 831 patients with solitary schwannomas. The trend line is a normal curve. B: Sex ratios are significantly different among the 3 groups (chi-square test, $p=0.012$ ). Asterisk indicates that the sex ratio of patients with schwannomatosis (Sch) is significantly different from that of patients with solitary schwannomas (chi-square test; solitary vs schwannomatosis, $p=0.003$; schwannomatosis vs NF2, $p=0.042$; solitary vs NF2, $p=0.924$ ). Because the chi-square test was performed 4 times, a $p$ value $<0.013$ was considered statistically significant. C: The mean ages at the first operation for spinal schwannomas are significantly different among the 3 groups (1-way ANOVA, $p<0.001$ ). Asterisk indicates that the mean age of NF2 is significantly different from that of patients with solitary schwannomas and schwannomatosis (post hoc Scheffe test; solitary vs NF2, $p<0.001$; schwannomatosis vs NF2, $p<0.001$; solitary vs schwannomatosis, $p=0.900)$. 


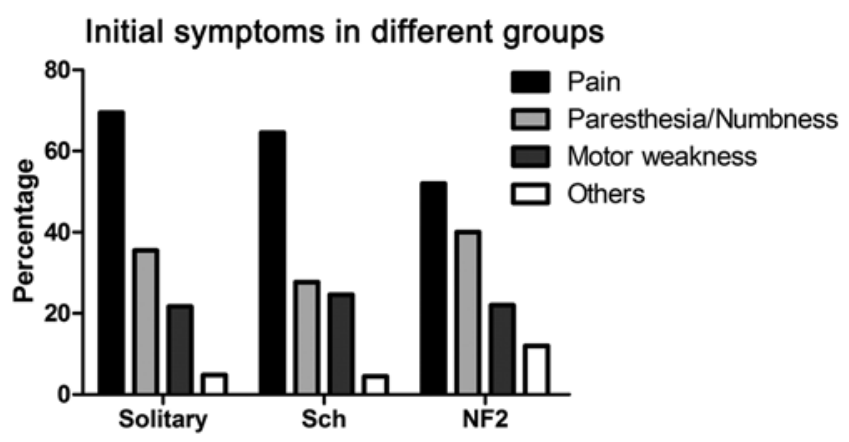

Initial symptoms in different spinal areas

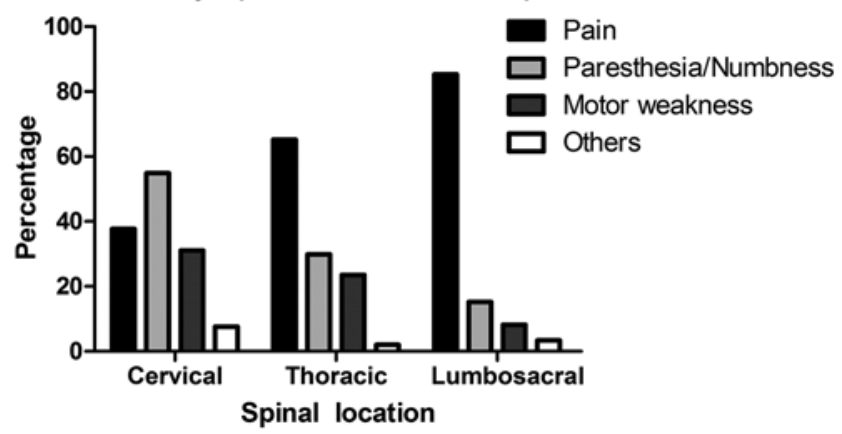

FIG. 2. Bar graphs depicting initial symptoms in different patient groups and spinal areas. Upper: Distribution of initial symptoms in patients with solitary schwannomas, schwannomatosis (Sch), and NF2. Lower: Distribution of initial symptoms in cervical, thoracic, and lumbosacral areas of patients with solitary schwannomas.

$p=0.001$; solitary vs. NF2, $p=0.011$; Fig. 3). Because spinal meningiomas and schwannomas were difficult to radiologically distinguish in NF2, they were both included in the analysis. Spinal tumors in NF2 and schwannomatosis could be grouped together; it was difficult to calculate the exact number of tumors. Thus, we calculated the spinal segments involved; each segment was assigned an equal value of $1 / 2$ (Fig. 4). We only calculated the segments that had tumors larger than $5 \mathrm{~mm}$ in diameter. Of 831 patients with solitary schwannomas, 343 tumors $(41.3 \%)$ were located in the cervical area, $221(26.6 \%)$ in the thoracic area, and 267 (32.1\%) in the lumbosacral area; the peak of distribution was at C1-3 and T12-L3 (Fig. 3A). Of 53 patients with NF2 with whole-spine MR images, a total of 151.5 segments were involved, including 42.8 (28.3\%) cervical segments, $49.2(32.5 \%)$ thoracic segments, and $59.5(39.3 \%)$ lumbosacral segments; a slight prominence in the lumbar area could be observed (Fig. 3B). Of 38 patients with schwannomatosis who underwent whole-spine MRI, 138 segments were involved, including 16 (11.6\%) cervical segments, 43.5 (31.5\%) thoracic segments, and $78.5(56.9 \%)$ lumbosacral segments. The peak of distribution was at T12-L5 (Fig. 3C). In patients with NF2 and schwannomatosis, the most common location of spinal schwannomas at the first operation was consistent with the distribution of their total spinal schwannomas (Fig. 3D). Of the first operations performed in 50 patients with NF2 who had undergone surgery, $23(45.1 \%)$ tumors were in the cervical area, $14(27.5 \%)$ were in the thoracic area, and $14(27.5 \%)$ were in the lumbosacral area (chi-square test, $\mathrm{p}=0.078$; compared with the total spinal distribution in
NF2). Because schwannomas in different areas could be resected in a single operation, the sum of schwannomas at the first operation was greater than 50. Of the first operations performed in 65 patients with schwannomatosis, 11 $(15.7 \%)$ tumors were in the cervical area, $22(31.4 \%)$ were in the thoracic area, and 37 (52.9\%) were in the lumbosacral area (chi-square test, $\mathrm{p}=0.680$, compared with the total spinal distribution in schwannomatosis).

\section{Patient Outcome}

Gross-total resection was achieved in 780 (93.9\%) patients with solitary schwannomas, $44(88 \%)$ with NF2, and $60(92.3 \%)$ with schwannomatosis. The patients in the 3 groups obtained similar benefits from the operation (chisquare test, $p=0.290$ ). Among 831 patients with solitary schwannomas, the result of surgical intervention was recovery (no evident symptoms or signs) in 416 (50.1\%), improved in 250 (30.1\%), stable in 144 (17.3\%), and worsened in $21(2.5 \%)$. Among the 50 patients with NF2 who underwent surgery, the result of surgical intervention was recovery in 19 patients (38\%), improvement in 20 (40\%), stable in $10(20 \%)$, and worsened in $1(2 \%)$. Among 65 patients with schwannomatosis, the result of surgical intervention was recovery in 35 (53.9\%), improvement in 23 (35.4\%), stable in $5(7.7 \%)$, and worsened in 2 (3.1\%). The postoperative outcome appeared to correlate with the patient's preoperative neurological condition. Of 343 patients with solitary schwannomas with preoperative motor weakness, $256(74.6 \%)$ still had postoperative motor weakness somewhat. Of 352 patients with solitary schwannomas with preoperative paresthesia/numbness, 231 (65.6\%) still had some degree of postoperative paresthesia/numbness. All 6 patients with schwannomatosis and postoperative motor weakness had motor weakness before the operation.

The prognosis varied in the schwannomatosis spinal schwannomas. The median follow-up in schwannomatosis was 41 months (range 1 month-6 years). Up until the last date of follow-up, most patients with schwannomatosis $(81.5 \%)$ had undergone a single spinal operation; 12 patients (18.5\%) had undergone multiple spinal operations. According to the consensus criteria,${ }^{23}$ patients with schwannomatosis and schwannomas limited to 1 limb or 5 or fewer contiguous segments of the spine were diagnosed as having segmental schwannomatosis. In our cohort, patients with multiple spinal operations all had nonsegmental schwannomatosis; the clinical characteristics of these patients are summarized in Table 1. Multiple spinal operations were caused by newly developed schwannomas. The median interval of spinal operations was 6 years (range 2-22 years). The mean age at the first spinal operation in patients with multiple spinal operations was significantly younger than that in patients with a single spinal operation $(34.8 \pm 13.6$ years vs $46.5 \pm 13.4$ years, respectively; 2 -tailed t-test, $\mathrm{p}=$ $0.008)$. Having a family history of schwannomatosis had no impact on whether a person required multiple spinal operations. Therefore, patients with or without a family history of schwannomatosis had a similar chance of undergoing multiple spinal operations (3/8 vs 9/57; chi-square test, $\mathrm{p}=0.319$ ). Male and female patients also had a similar chance to undergo multiple spinal operations (6/47 vs. 6/18, respectively; chi-square test, $\mathrm{p}=0.120$ ). 

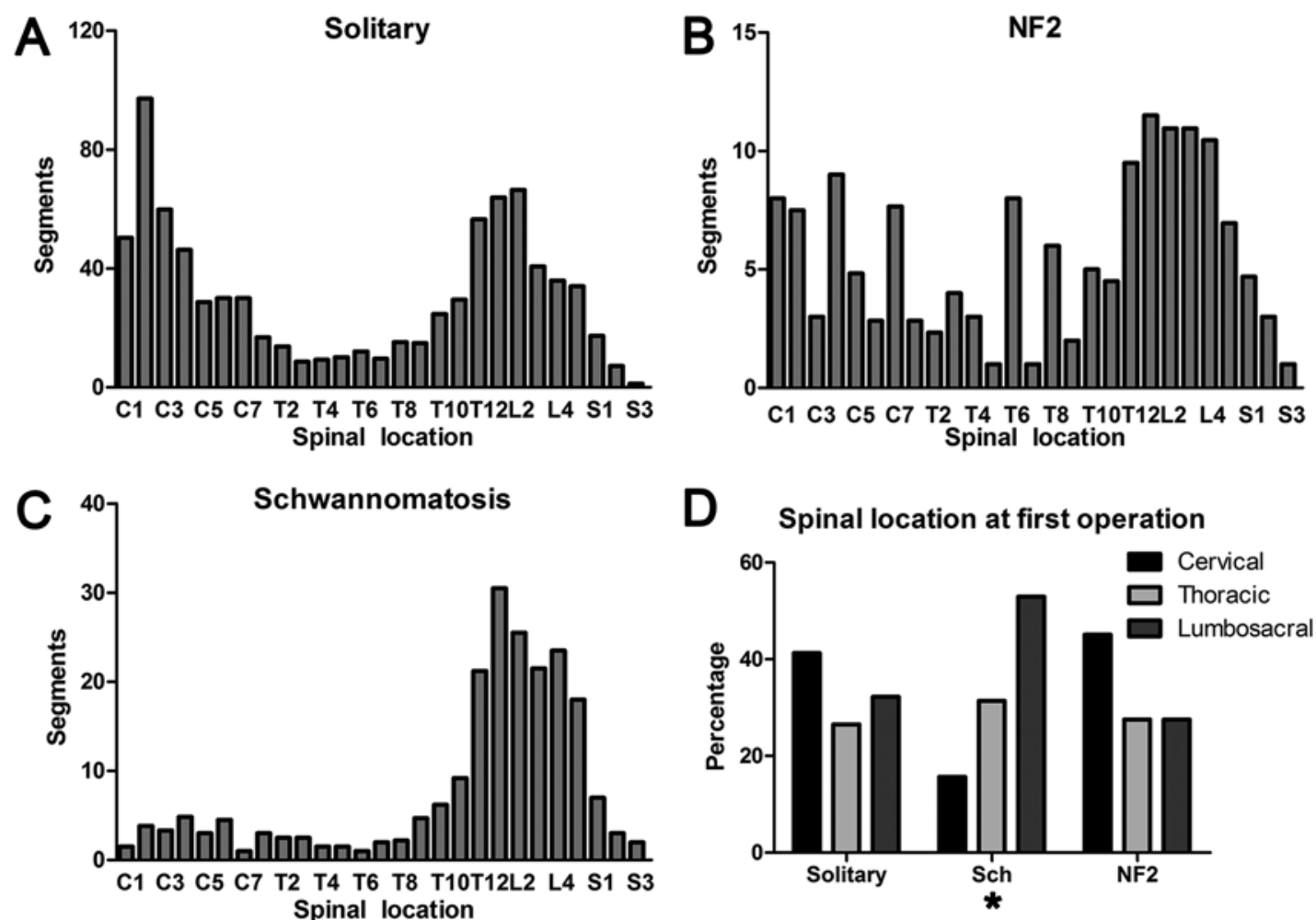

FIG. 3. Bar graphs depicting distribution of spinal tumors in the 3 groups. A: Distribution in patients with solitary schwannomas. B: Distribution of intradural-extramedullary tumors in patients with NF2. C: Distribution in patients with schwannomatosis. D: Distributions of spinal schwannomas at the first operation in the 3 groups.

\section{Multiple Small Cauda Equina Nodules}

Multiple small cauda equina nodules were common in our patients with NF2 and schwannomatosis. Of 84 patients with NF2 who underwent lumbar spine MRI, 73 (86.9\%) developed these lesions; the nodules of $13(15.5 \%)$ patients were small in number (less than 5), and those of $60(71.4 \%)$ patients were large in number (more than 5; Fig. 5). Of 60 patients with schwannomatosis who underwent lumbar spine MRI, 28 (46.7\%) developed these lesions; the nodules of $11(18.3 \%)$ patients were small in number (less than
5), and those of 17 (28.3\%) patients were large in number (more than 5). Multiple cauda equina nodules in 6 patients with NF2 were followed up using MRI (median 1.5 years, range 8 months -3 years). Almost all nodules remained static in size and only showed imaging-based progression.

\section{Other Characteristics of Schwannomatosis}

Of 65 patients with schwannomatosis, 28 (43.1\%) had schwannomas outside the spinal canal, and $37(56.9 \%)$ did not. Of 51 patients who underwent cranial MRI, $5(9.8 \%)$
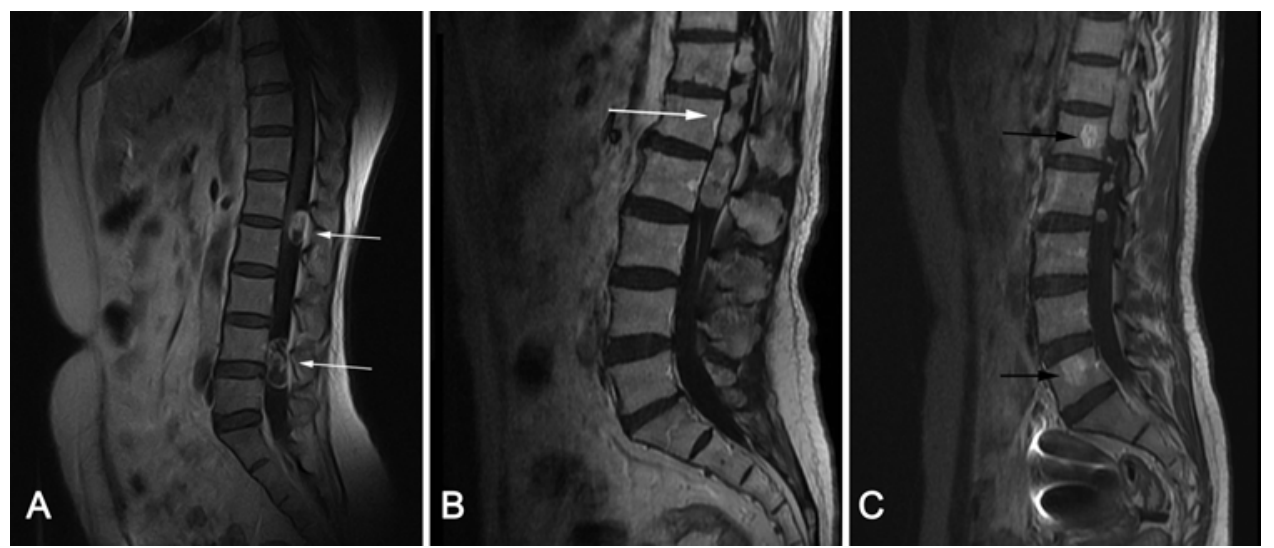

FIG. 4. Sagittal MRI (A and B) demonstrating how the spinal segments are calculated, and enhanced MRI (C) showing vertebral hemangiomas. A: Two spinal schwannomas are located at L1-L2 and L4-L5 (arrows). The spinal segments of L-1, L-2, L-4, and $\mathrm{L}-5$ are all valued as 1/2. B: Multiple spinal schwannomas group together at T12-L2 (arrow). The spinal segments of T-12, $\mathrm{L}-1$, and L-2 are all valued as 1. C: MR image demonstrating 2 enhanced lesions that are likely hemangiomas (arrows) in L-5 and L-1 vertebral bodies. 
TABLE 1. Summary of characteristics in 12 patients with schwannomatosis who underwent multiple spinal operations

\begin{tabular}{|c|c|c|c|c|c|c|c|c|}
\hline \multirow{2}{*}{$\begin{array}{l}\text { Case } \\
\text { No. }\end{array}$} & \multirow[b]{2}{*}{ Sex } & \multicolumn{2}{|c|}{ Age (yrs) } & \multirow[b]{2}{*}{ Cranial MRI } & \multirow{2}{*}{$\begin{array}{l}\text { Location of Spinal } \\
\text { Schwannomas }\end{array}$} & \multirow{2}{*}{$\begin{array}{l}\text { No. of } \\
\text { Ops }\end{array}$} & \multirow{2}{*}{$\begin{array}{c}\text { Mean Interval } \\
\text { of Ops (yrs) }\end{array}$} & \multirow[b]{2}{*}{ Current Status } \\
\hline & & Now & 1 st Op & & & & & \\
\hline $1^{*}$ & M & 52 & 38 & Normal & T12-S2 & 3 & 4.5 & Numbness in both legs \\
\hline $2 \dagger$ & $\mathrm{F}$ & 48 & 40 & Meningioma & C5-6, T3-4, T-5, L2-S1 & 2 & 8 & No symptoms \\
\hline $3 \dagger$ & M & 70 & 55 & NA & C3-4, L-2, L4-5 & 2 & 14 & Numbness in rt leg \\
\hline $4^{*}$ & M & 57 & 42 & Normal & T1-2, L-4, L5-S1 & 3 & 4.5 & Numbness in rt leg \\
\hline $5^{*}$ & $\mathrm{~F}$ & 56 & 40 & TS & C2-3, L1-S1 & 2 & 16 & Voiding difficulty \\
\hline $6^{*}$ & $\mathrm{~F}$ & 50 & 23 & Normal & T5-7, L1-2 & 2 & 22 & Weakness in both legs \\
\hline $7^{*}$ & $\mathrm{~F}$ & 23 & 10 & Normal & C6-7, T3-4, T-9, T12-L1, L3-4 & 6 & 2.6 & No symptoms \\
\hline $8^{*}$ & $\mathrm{~F}$ & 63 & 57 & NA & C3-5, T-1, T-8, T11-12 & 2 & 6 & Chronic pain in rt arm \& leg \\
\hline $9 \dagger$ & M & 62 & 34 & CS & C-2, T-1, T-9, L-1 & 2 & 22 & Weakness in both legs \\
\hline $10^{*}$ & $\mathrm{~F}$ & 29 & 21 & Normal & L1-2, L3-4, L4-5 & 3 & 3.5 & Numbness in rt leg \\
\hline $11^{*}$ & M & 46 & 29 & NA & C2-3, T12-L1, L-3 & 3 & 4.5 & Numbness in rt arm \& It leg \\
\hline $12^{*}$ & M & 44 & 29 & Normal & T4-5, T11-12, L4-5, S-1 & 2 & 10 & Weakness in It leg \\
\hline
\end{tabular}

$\mathrm{CS}=$ cerebellar schwannoma; $\mathrm{NA}=$ not available.

* Sporadic schwannomatosis.

$\dagger$ Familial schwannomatosis.

had cranial schwannomas, including 3 unilateral trigeminal schwannomas (TSs), 1 hypoglossal nerve schwannoma, and 1 schwannoma located in the cerebellar hemisphere. Schwannomas of $22(33.8 \%)$ patients were limited to 5 or fewer contiguous spinal segments; they were diagnosed as segmental schwannomatosis according to the consensus criteria. ${ }^{23}$ No significant difference could be found in the mean age at the first spinal operation of patients with segmental and nonsegmental schwannomas $(42.3 \pm 14.4$ vs $48.6 \pm 12.8$, respectively; 2 -tailed t-test, $\mathrm{p}=0.089$ ). A family history of schwannomatosis was present in $8(12.3 \%)$ individuals, each from a different family. No significant difference could be found in mean age at the first spinal operation of patients with and without a family history $(42.5 \pm 10.3$ vs $44.6 \pm 14.6$, respectively; 2 -tailed t-test, $p$ $=0.690)$. Nineteen of the 65 patients had no children; the remaining 46 patients had 68 children. The median age of the offspring was 28 years (range 3-50 years). Of the 68 offspring, only 2 from different families had suspicious subcutaneous schwannomas.

\section{Coexisting Conditions of Schwannomatosis}

Fourteen (21.5\%) patients with schwannomatosis had various types of cysts diagnosed clinically, pathologically, or radiographically. Seven (10.8\%) patients had radiographically diagnosed asymptomatic vertebral hemangiomas (Fig. 4C). Six (9.2\%) patients had pathologically confirmed lipomas. Of the 18 female patients with schwannomatosis, $3(16.7 \%)$ had pathologically confirmed uterine myomas. Some patients also had other conditions, including meningiomas $(n=3)$, polyps $(n=3)$, thyroid fibromas $(n=3)$, inguinal hernia $(\mathrm{n}=2)$, nasal angioma $(\mathrm{n}=1)$, pituitary tumor $(\mathrm{n}=1)$, parotid tumor $(\mathrm{n}=1)$, bladder cancer $(\mathrm{n}=1)$, and optic atrophy $(\mathrm{n}=1)$.

\section{Discussion}

Schwannomatosis is the third major form of neurofibromatosis. Unlike NF1 and NF2, the definite causative gene of schwannomatosis remains undetermined. Germline mutations of SMARCB1 and LZTR1 have been found
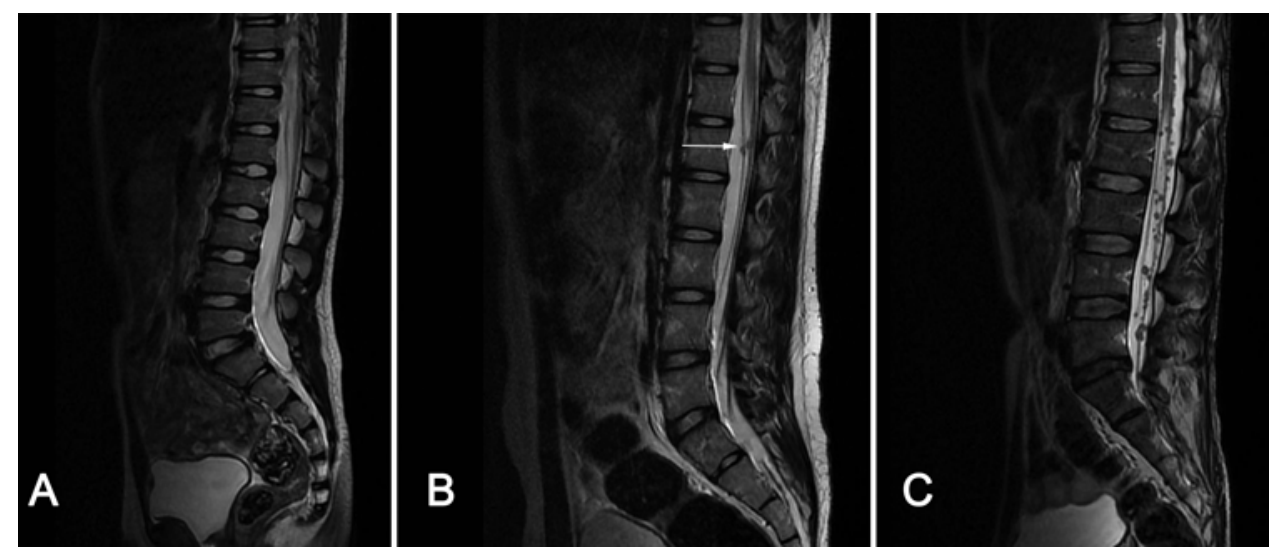

FIG. 5. Sagittal T2-weighted MR images demonstrating multiple hypointense, small, cauda equina nodules in patients with NF2 and schwannomatosis (each only a few millimeters in size): A: Normal cauda nerve. B: A small number of small cauda equina nodules (<5, arrow). C: A large number of small cauda equina nodules $(>5)$. 
in some patients with schwannomatosis. ${ }^{16,29}$ However, constitutional mutations in the SMARCBI gene have only occurred in $40 \%-50 \%$ of familial cases and $8 \%-10 \%$ of sporadic cases of schwannomatosis. ${ }^{30}$ The acute incidence of constitutional mutations of the LZTRI gene in schwannomatosis remains unclear. Because the phenotype of schwannomatosis overlaps with that of NF2, the main challenge in diagnosing schwannomatosis is to differentiate it from NF2. The most critical diagnostic criteria for schwannomatosis are the revised clinical criteria. ${ }^{3}$ However, they still cannot rule out all patients with mosaic NF2, because these patients can manifest multiple nonvestibular schwannomas, and their blood samples can be free of NF2 mutations. Detecting the mutations of the $N F 2$ gene in a single tumor cannot help to differentiate schwannomatosis from NF2, because mutations of the NF2 gene are also common in tumors of patients with schwannomatosis. ${ }^{17,34}$ Mutations of the NF2 gene in schwannomatosis are believed to be caused by a "four-hit mechanism,"14,34 types of mutations in different tumors should be random. Plotkin et al. ${ }^{30}$ suggested detecting NF2 mutations in 2 or more separate tumors of patients with schwannomatosis. If the types of mutations are different, NF2 can be ruled out. ${ }^{20}$ Detecting mutations of SMARCBI and LZTRI can also help with the diagnosis of schwannomatosis. Additionally, it is very difficult to definitively diagnose 1 patient with schwannomatosis. We cannot guarantee that our schwannomatosis cohort did not contain some patients with mosaic or mild-phenotype NF2, but these patients with NF2 should be very small in number, and they should not affect the statistical results in our large cohort.

In schwannomatosis, no known predisposition exists regarding race or sex. ${ }^{26}$ However, in our schwannomatosis cohort, we noticed a higher prevalence in male patients $(72.3 \%)$ than in female patients $(27.7 \%$; Fig. 1B). Whether male patients with schwannomatosis have a higher prevalence of spinal schwannomas remains to be studied. Previous reports have indicated that symptomatic spinal schwannomas in NF2 tend to grow faster, more likely infiltrating nerve roots and progressing to serious deficits sooner. ${ }^{21}$ In our cohort, we also found that patients with NF2 were the youngest at the first spinal schwannoma operation and had the shortest duration of preoperative symptoms. Patients with schwannomatosis appeared to have a similar clinical course as patients with solitary schwannomas. Chen et al. ${ }^{6}$ and Seppälä et al. ${ }^{33}$ also found that patients with schwannomatosis were similar in age at operation as patients with solitary schwannomas. However, Gonzalvo et al..$^{13}$ found that the patient age at onset of schwannomatosis tended to be younger than that of solitary schwannomas. These previous studies included both the CNS and peripheral schwannomas; our study focused on spinal schwannomas.

The distribution of spinal schwannomas varied among the 3 groups (Fig. 2). The most common location of solitary spinal schwannoma was controversial in previous studies, $5,7,18,19,31,32$ and conflicting evidence supported a predilection for the cervical, lumbosacral, and thoracic spine. These studies were usually relatively small in size. In our largest cohort, we observed the most common location in patients with solitary spinal schwannomas to be at the high cervical and thoracolumbar segments. A previous study indicated that intradural-extramedullary NF2 tumors did not have a preferred spinal location. ${ }^{25}$ In our NF2 cohort, we obtained the same result and could only observe a slight predominance in the lumbar area. As reported ${ }^{26}$ spinal schwannomas in our patients with schwannomatosis were most common in the lumbar area. Different distributions of spinal schwannomas in the 3 groups might be caused by different etiologies. Knowing these distribution patterns can help us educate patients with schwannomatosis to recognize early signs and symptoms of spinal schwannomas.

In our cohort, the patients in the 3 groups developed similar initial symptoms; pain was the most common. Previous reports also indicate that the most common symptom in schwannomatosis is pain. ${ }^{13,26}$ Symptoms of pain in schwannomatosis might be difficult to treat. Merker et al. ${ }^{26}$ reported that local pain in schwannomatosis could be completely relieved in less than half of the surgeries. In our cohort, 42 patients developed preoperative pain, most patients were free of the pain after the operation, 6 patients were partially relieved, and only 1 was unchanged. Previous reports were mainly focused on peripheral schwannomas. Our study suggests that pain caused from spinal schwannomas in schwannomatosis can be treated successfully with surgery.

Most spinal schwannomas in patients with non-NF can be safely resected by experienced surgeons. ${ }^{31}$ Klekamp et al. ${ }^{21}$ reported that 17 patients with NF2 showed little improvement after the spinal schwannoma operation. In our cohort, the patients in the 3 groups obtained similar benefits from the spinal operation. Most spinal schwannomas of our patients with schwannomatosis were resected safely. During the operation, we found that some spinal schwannomas in schwannomatosis had characteristics that were similar to those of NF2 tumors: $:^{21}$ the tumors demonstrated tight adherence to the nerve roots; tumors grew along the root over long distances; multiple tumors were carried by a single nerve root; and multiple tumors could be grouped together to appear as 1 single tumor on MR images. Because of these characteristics, it was difficult to totally resect tumors without causing neurological deficits. On the other hand, the patient's preoperative condition correlated with the surgical outcome. ${ }^{32}$ In our cohort, we found that patients with preoperative motor weakness/paresthesia were more likely to have postoperative motor weakness/ paresthesia. Thus, to obtain satisfactory treatment outcomes, surgery should be performed quickly before spinal schwannomas cause serious neurological deficits.

The prognosis varied among the spinal schwannomas of our patients with schwannomatosis. Most patients with schwannomatosis $(81.5 \%)$ underwent a single spinal operation; other patients (18.5\%) underwent multiple spinal operations. These variations in schwannomatosis have also been reported in several previous studies; ${ }^{13,33}$ some patients may develop new symptomatic tumors every few years, while others do not. In our cohort, patients with nonsegmental schwannomatosis or patients with early onset appeared to have a poor prognosis and were more likely to undergo multiple spinal operations. However, family history and sex did not affect the prognosis.

Small cauda equina nodules, which were most likely small schwannomas, were common in our patients with 
schwannomatosis (46.7\%) and NF2 (86.9\%). They were usually small in size and might not cause evident symptoms. If these small cauda equina nodules progress into large tumors, they will be very difficult to treat with surgery. A previous study indicated that most patients with preoperative autonomic deficits caused by cauda equina tumors did not recover significantly from their bladder and bowel disturbances after surgical management. ${ }^{31}$ In our cohort, 1 patient with multiple cauda equina schwannomas had voiding difficulty after the spinal operation (Case 5, Table 1). Several previous reports have indicated that these small tumors in NF2 had a relatively static behavior and could be stable for a long time without progression..${ }^{1,9}$ Dow et al. ${ }^{9}$ speculated that these multiple small tumors in NF2 might have a chance to represent hamartomas without the propensity to grow. Instead, large extramedullary tumors $(>5 \mathrm{~mm})$ in NF2 were more likely to progress. ${ }^{9}$ Ozawa et al. ${ }^{27}$ reported that the natural history of spinal schwannoma could have several growth patterns: unchanging, continuous slight growth, and initial slight growth followed by rapid growth. These small cauda equina schwannomas may present with an unchanging pattern. Because most patients with NF2 have significant problems related to bilateral vestibular schwannomas as well as other intracranial neoplasms, a conservative approach is usually used to manage these small spinal tumors. With the improvement in treatment strategies, the life expectancy of patients with NF2 can be extended. Additionally, the life span of patients with schwannomatosis is usually not affected. ${ }^{36}$ It is very essential to know the long-term biological patterns of these small cauda equina nodules.

The indication for the excision of schwannomas in patients with schwannomatosis usually follows the same principles as the management of sporadic schwannomas. ${ }^{13}$ Symptomatic tumors or those demonstrating enlargement during follow-up could be resected. Routine whole-spine MRI should be performed on every patient with schwannomatosis. Like NF2, new tumors can develop in patients with schwannomatosis. Asymptomatic tumors can be monitored conservatively by serial MRI studies, usually at a yearly interval. ${ }^{15}$ However, waiting for patients to experience symptoms clearly confers a delay on time to surgery, which may inevitably reduce the chances of achieving a favorable outcome. Up until the last date of follow-up, all patients with segmental schwannomatosis in our cohort underwent a single spinal operation. It seemed appropriate to perform early operations on these patients. On the other hand, there might be a concern for malignant transformation during follow-up. A previous report has indicated the risk for malignancy in patients with schwannomatosis, ${ }^{13}$ but it is not common. We did not find any malignant schwannomas in our cohort. Merker et al. ${ }^{26}$ reported that some tumors in schwannomatosis might be misdiagnosed as malignant schwannomas on initial pathological review; therefore, a diagnosis of malignant schwannoma should be viewed with caution.

Schwannomatosis is an inherited disease. Genetic counseling is essential for patients with schwannomatosis. Unlike with NF1 and NF2, schwannomatosis is sporadic; only approximately $15 \%-25 \%$ of cases are inherited from an affected parent. ${ }^{30}$ In our cohort, 8 (12.3\%) individuals had a family history of schwannomatosis. Clear instances of autosomal dominant transmission in schwannomatosis have been reported..$^{24}$ The transmission risk can be assumed to be $50 \%$ in familial schwannomatosis; however, the risks for a sporadic case are less clear. ${ }^{30}$ Nonpenetrance has been described in some patients with schwannomatosis.$^{29}$ In our cohort, the transmission risk was quite low (2/68), but whole-body MRI was not performed on these offspring. Some asymptomatic tumors might be missed, and some offspring might still be too young to experience symptoms.

Cranial schwannomas are not common in patients with schwannomatosis. ${ }^{26}$ In our cohort, $5(9.8 \%)$ patients with schwannomatosis had cranial nonvestibular schwannomas, which were all solitary. Unilateral vestibular schwannoma has been reported in schwannomatosis. Some researchers believe that unilateral vestibular schwannoma should not be an exclusion criterion for the clinical diagnosis of schwannomatosis. ${ }^{35}$ However, patients with suspicious sporadic schwannomatosis with unilateral vestibular schwannoma should be cautioned about the possibility of having NF2. In fact, according to Manchester diagnostic criteria, ${ }^{4}$ patients with unilateral vestibular schwannoma and 2 or more other schwannomas can be diagnosed as NF2. One patient in our cohort with NF2 had unilateral vestibular schwannoma and multiple spinal schwannomas; heterozygous deletion of whole exon 11 in NF2 was identified in his lymphocyte DNA. Moreover, patients with multiple cranial nonvestibular schwannomas should also be cautioned about NF2, especially when a patient has schwannomas located at the cerebellopontine angle. One 30 -year-old man in our NF2 cohort had suspicious bilateral TSs, and his hearing ability was not affected (Fig. 6). Heterozygous nonsense NF2 mutation, c.439 C > T in exon 4 , was identified in his lymphocyte DNA.

In our cohort, patients with schwannomatosis had various coexisting conditions. High rates of cysts (60\%) and lipomas (13\%) in schwannomatosis have also been published in a previous report. ${ }^{26}$ Moreover, we found a high rate of asymptomatic vertebral hemangiomas (10.8\%) in our patients with schwannomatosis (Fig. 5C); however, the rate was similar to the incidence of vertebral hemangiomas in the general population $(10 \%-12 \%) .^{12}$ The co-occur-
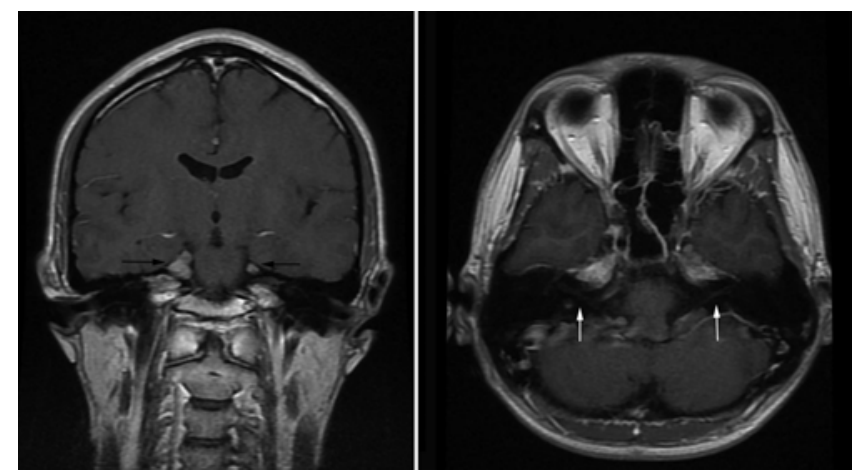

FIG. 6. MRI demonstrating suspicious bilateral TSs in 1 patient with NF2. Left: Coronal cranial MRI demonstrating enhanced lesions at bilateral cerebellopontine angles (black arrows). Right: Axial cranial MRI demonstrating that the bilateral acoustic nerves of the patient are normal (white arrows). 
rence of these 2 anomalies was likely to be coincidental. Meningiomas are not common in schwannomatosis. ${ }^{26}$ In our schwannomatosis cohort, 2 patients had cranial meningiomas, and 1 patient had spinal meningioma, which were all solitary. Although patients with schwannomatosis can have multiple meningiomas, ${ }^{2,26}$ according to Manchester diagnostic criteria, patients with multiple meningiomas and 2 or more schwannomas can be diagnosed as having NF2, making Manchester diagnostic criteria the most sensitive criteria for NF2 ${ }^{4}$ One can never be too cautious when diagnosing schwannomatosis. Because patients with NF2 and their offspring usually need more intensive follow-up than patients with schwannomatosis, a delayed diagnosis may cause serious problems. ${ }^{10,28}$

\section{Limitations}

The main limitation of this study is its retrospective nature and single-institution basis. The blood samples of many patients with schwannomatosis were not available to exclude constitutional NF2 mutations. The duration of follow-up was too short to analyze the recurrence rates of spinal schwannomas. A multicenter, long-term prospective study is needed to gather more comprehensive information concerning spinal schwannomas in schwannomatosis.

\section{Conclusions}

The clinical features of spinal schwannomas vary among patients with solitary schwannomas, NF2, and schwannomatosis. Spinal schwannomas of NF2 appear to be more aggressive than those in patients with solitary schwannomas and schwannomatosis. Spinal schwannomas of schwannomatosis are predominant in the lumbar area, and most of them can be treated successfully with surgery. The prognosis varies among spinal schwannomas of schwannomatosis; some patients may need multiple operations due to newly developed schwannomas. Sometimes, it is difficult to differentiate schwannomatosis from NF2 based on clinical manifestations. It is prudent to perform close follow-up in patients and their offspring when undetermined schwannomatosis is detected.

\section{References}

1. Aboukais R, Baroncini M, Zairi F, Bonne NX, Schapira S, Vincent $C$, et al: Prognostic value and management of spinal tumors in neurofibromatosis type 2 patients. Acta Neurochir (Wien) 155:771-777, 2013

2. Bacci C, Sestini R, Provenzano A, Paganini I, Mancini I, Porfirio B, et al: Schwannomatosis associated with multiple meningiomas due to a familial SMARCB1 mutation. Neurogenetics 11:73-80, 2010

3. Baser ME, Friedman JM, Evans DG: Increasing the specificity of diagnostic criteria for schwannomatosis. Neurology 66:730-732, 2006

4. Baser ME, Friedman JM, Wallace AJ, Ramsden RT, Joe H, Evans DG: Evaluation of clinical diagnostic criteria for neurofibromatosis 2. Neurology 59:1759-1765, 2002

5. Celli P, Trillò G, Ferrante L: Spinal extradural schwannoma. J Neurosurg Spine 2:447-456, 2005

6. Chen SL, Liu C, Liu B, Yi CJ, Wang ZX, Rong YB, et al: Schwannomatosis: a new member of neurofibromatosis family. Chin Med J (Engl) 126:2656-2660, 2013

7. Conti P, Pansini G, Mouchaty H, Capuano C, Conti R: Spinal neurinomas: retrospective analysis and long-term outcome of
179 consecutively operated cases and review of the literature. Surg Neurol 61:34- 44, 2004

8. Daras M, Koppel BS, Heise CW, Mazzeo MJ, Poon TP, Duffy KR: Multiple spinal intradural schwannomas in the absence of von Recklinghausen's disease. Spine (Phila Pa 1976) 18:2556-2559, 1993

9. Dow G, Biggs N, Evans G, Gillespie J, Ramsden R, King A: Spinal tumors in neurofibromatosis type 2. Is emerging knowledge of genotype predictive of natural history? J Neurosurg Spine 2:574-579, 2005

10. Evans DG: Neurofibromatosis type 2 (NF2): a clinical and molecular review. Orphanet J Rare Dis 4:16, 2009

11. Evans DG, Huson SM, Donnai D, Neary W, Blair V, Newton V, et al: A clinical study of type 2 neurofibromatosis. Q J Med 84:603-618, 1992

12. Fox MW, Onofrio BM: The natural history and management of symptomatic and asymptomatic vertebral hemangiomas. J Neurosurg 78:36-45, 1993

13. Gonzalvo A, Fowler A, Cook RJ, Little NS, Wheeler H, McDonald KL, et al: Schwannomatosis, sporadic schwannomatosis, and familial schwannomatosis: a surgical series with long-term follow-up. Clinical article. J Neurosurg 114:756762,2011

14. Hadfield KD, Newman WG, Bowers NL, Wallace A, Bolger C, Colley A, et al: Molecular characterisation of SMARCB1 and NF2 in familial and sporadic schwannomatosis. J Med Genet 45:332-339, 2008

15. Huang JH, Simon SL, Nagpal S, Nelson PT, Zager EL: Management of patients with schwannomatosis: report of six cases and review of the literature. Surg Neurol 62:353-361, 2004

16. Hulsebos TJ, Plomp AS, Wolterman RA, Robanus-Maandag EC, Baas F, Wesseling P: Germline mutation of INI1/ SMARCB1 in familial schwannomatosis. Am J Hum Genet 80:805-810, 2007

17. Jacoby LB, Jones D, Davis K, Kronn D, Short MP, Gusella J, et al: Molecular analysis of the NF2 tumor-suppressor gene in schwannomatosis. Am J Hum Genet 61:1293-1302, 1997

18. Jeon JH, Hwang HS, Jeong JH, Park SH, Moon JG, Kim CH: Spinal schwannoma; analysis of 40 cases. J Korean Neurosurg Soc 43:135-138, 2008

19. Jinnai T, Koyama T: Clinical characteristics of spinal nerve sheath tumors: analysis of 149 cases. Neurosurgery 56:510515,2005

20. Kashima TG, Gibbons MR, Whitwell D, Gibbons CL, Bradley KM, Ostlere SJ, et al: Intraosseous schwannoma in schwannomatosis. Skeletal Radiol 42:1665-1671, 2013

21. Klekamp J, Samii M: Surgery of spinal nerve sheath tumors with special reference to neurofibromatosis. Neurosurgery 42:279-290, 1998

22. Landi A, Dugoni DE, Marotta N, Mancarella C, Delfini R: Spinal schwannomatosis in the absence of neurofibromatosis: A very rare condition. Int J Surg Case Rep 2:36-39, 2011

23. MacCollin M, Chiocca EA, Evans DG, Friedman JM, Horvitz R, Jaramillo D, et al: Diagnostic criteria for schwannomatosis. Neurology 64:1838-1845, 2005

24. MacCollin M, Willett C, Heinrich B, Jacoby LB, Acierno JS $\mathrm{Jr}$, Perry A, et al: Familial schwannomatosis: exclusion of the NF2 locus as the germline event. Neurology 60:1968-1974, 2003

25. Mautner VF, Tatagiba M, Lindenau M, Fünsterer C, Pulst SM, Baser ME, et al: Spinal tumors in patients with neurofibromatosis type 2: MR imaging study of frequency, multiplicity, and variety. AJR Am J Roentgenol 165:951-955, 1995

26. Merker VL, Esparza S, Smith MJ, Stemmer-Rachamimov A, Plotkin SR: Clinical features of schwannomatosis: a retrospective analysis of 87 patients. Oncologist 17:1317-1322, 2012 
27. Ozawa H, Onoda Y, Aizawa T, Nakamura T, Koakutsu T, Itoi E: Natural history of intradural-extramedullary spinal cord tumors. Acta Neurol Belg 112:265-270, 2012

28. Pérez-Grau M, Miró N, Prades J, Vergés J, Lareo S, RocaRibas F: [Neurofibromatosis type 2.] Acta Otorrinolaringol Esp 61:306-311, 2010 (Span)

29. Piotrowski A, Xie J, Liu YF, Poplawski AB, Gomes AR, Madanecki P, et al: Germline loss-of-function mutations in LZTR1 predispose to an inherited disorder of multiple schwannomas. Nat Genet 46:182-187, 2014

30. Plotkin SR, Blakeley JO, Evans DG, Hanemann CO, Hulsebos TJ, Hunter-Schaedle K, et al: Update from the 2011 International Schwannomatosis Workshop: From genetics to diagnostic criteria. Am J Med Genet A 161A:405-416, 2013

31. Safavi-Abbasi S, Senoglu M, Theodore N, Workman RK, Gharabaghi A, Feiz-Erfan I, et al: Microsurgical management of spinal schwannomas: evaluation of 128 cases. J Neurosurg Spine 9:40-47, 2008

32. Seppälä MT, Haltia MJ, Sankila RJ, Jääskeläinen JE, Heiskanen O: Long-term outcome after removal of spinal schwannoma: a clinicopathological study of 187 cases. J Neurosurg 83:621-626, 1995

33. Seppälä MT, Sainio MA, Haltia MJ, Kinnunen JJ, Setälä KH, Jääskeläinen JE: Multiple schwannomas: schwannomatosis or neurofibromatosis type 2? J Neurosurg 89:36-41, 1998

34. Sestini R, Bacci C, Provenzano A, Genuardi M, Papi L: Evidence of a four-hit mechanism involving SMARCB1 and NF2 in schwannomatosis-associated schwannomas. Hum Mutat 29:227-231, 2008

35. Smith MJ, Kulkarni A, Rustad C, Bowers NL, Wallace AJ,
Holder SE, et al: Vestibular schwannomas occur in schwannomatosis and should not be considered an exclusion criterion for clinical diagnosis. Am J Med Genet A 158A:215-219, 2012

36. Uhlmann EJ, Plotkin SR: Neurofibromatoses. Adv Exp Med Biol 724:266-277, 2012

\section{Disclosure}

This study was supported by the National Science and Technology Support Program of the 12th Five-Year of China (grant no. 2012BAI12B03) and Natural Science Foundation of Beijing (grant no. 7112049).

\section{Author Contributions}

Conception and design: Liu, Li. Acquisition of data: Li, Zhao, Zhang, Z Wang, X Wang, Z Yang, J Yang. Analysis and interpretation of data: Li, Zhang, Z Wang, X Wang, B Wang, Z Yang, J Yang, Gao. Drafting the article: Li, X Wang. Critically revising the article: Liu, Zhao. Reviewed submitted version of manuscript: all authors. Approved the final version of the manuscript on behalf of all authors: Liu. Statistical analysis: Zhang, Z Wang, X Wang, B Wang, Z Yang. Administrative/technical/material support: Gao. Study supervision: Liu, Zhao, J Yang, Gao.

\section{Correspondence}

Pinan Liu, Department of Neurosurgery, Beijing Tian Tan Hospital, Capital Medical University, 6 Tiantan Xili, Dongcheng District, Beijing100050, China. email: pinanliu@ccmu.edu.cn. 